\title{
Multipath Routing Scheme for Industrial Real-time Wireless Sensor Networks
}

\author{
Hai-Jiang Hu, Shu-Qin Liu, Yi-Fan Wang, Ju-Ju Hu \\ College of Physics and Communication Electronics, Jiangxi Normal University, Nanchang, China \\ E-mail: haijianghu@jxnu.edu.cn
}

\begin{abstract}
In the industrial scenes, the real-time property has to be considered in the data transmission. A novel routing scheme is proposed to reduce the packet loss rate effectively in the industrial wireless sensor networks. The scheme is based on Bellman-Ford algorithm, and saves some other shorter paths for the multipath transmission. According to different requirements of QoS in data transferring, the scheme can use more than one path to send the same packet synchronously. Moreover, the scheme use a unique label to mark every packet to assure that the receiver can discard the same packet from different path after a packet arrives in the receiver. Therefore, this scheme can reduce the probability of packet retransmission, and enhance the real-time property in the industrial wireless sensor networks.
\end{abstract}

Keywords-routing; bellman-ford algorithm; real-time transferring; wireless sensor networks

\section{INTRODUCTION}

Routing is one of the key technologies of networks. There are two main functions in the traditional routing protocols. One is to maintain and refresh the routing table; another is to calculate the shortest path (the smallest number of hops or time delay) based on the messages of routing table. However, besides the smallest time delay of data transferring, the low packet loss rate is also an important issue in the routing design for industrial real-time communication. If a packet is discarded in the process of transferring because of the quality of physical channels, data verification, or congestion control, the retransmission process will prolong the time delay. Therefore, there is a considerable problem how the routing algorithm decreases the influence of packet loss rate in the industrial communication. Some routing algorithms were proposed to solve the problem [2-12]. Most proposed algorithms focus on the choice of the shortest path based on the multi-restriction conditions. In the wireless sensor networks (WSN) [1], the routing is realized not only in the network layer, but also in the media access control (MAC). The routing in MAC is an important character in WSN. In industrial scene, the quality of the wireless channel is poorer than the wired channel. So it is necessary to avoid the packet loss in the routing design. A novel routing scheme is proposed to realize both the shortest path and the low packet loss rate. The scheme uses the redundancy transmission paths to reduce the rate of packet loss based on the improved Bellman-Ford algorithm.

\section{PRINCIPLE OF MULTIPATH ROUTING SCHEME}

The multipath routing scheme is based on Bellman-Ford algorithm [13], which computes single-source shortest paths in a weighted digraph. We define a weighted digraph as $G$ $(V, E)$, where $V$ and $E$ are the set of vertices and edges respectively. In $G$ we also define $S$ as source vertex, $n$ as the number of vertices, Weight $(u, v)$ as the route metrics from vertex $u$ to vertex $v$, Distance $(v)$ as the shortest path from $S$ to vertex $v, \operatorname{Predecessor}(v)$ as the next hop vertex from $v$, respectively.

After executing Bellman-Ford algorithm, we can judge whether the digraph $\mathrm{G}$ contains a negative cycle. If $G$ cannot contain the negative cycle, Distance $(v)$ is the shortest distance from $S$ to vertex $v$. The Bellman-Ford algorithm is often used in the routing designs, which consists of three steps. The first step is that each node calculates the distances between itself and all other nodes within the Autonomous system and stores this information as a table. The second step is that each node sends its table to all neighboring nodes. The third one is that a node calculates the shortest routes to all other nodes and updates its own table to reflect any changes when this node receives distance tables from its neighbors.

Bellman-Ford algorithm only refresh Distance (v) and Predecessor (v) in the every loop. If Distance (v) is not the shortest path, Distance (v) is replaced by the new the shortest path. Therefore, Bellman-Ford algorithm only calculates and saves the shortest path. However, in the industrial scenes, the packet retransmission after the packet loss is a major factor which increases the time delay of the data transmission. For this reason, we not only save the shortest path, but also save some other shorter paths for the multipath transmission in the Bellman-Ford algorithm. We can define a list PathList(v) which includes some valid paths from $S$ to vertex $v$. The pseudo codes of PathList(v) are defined as follows:

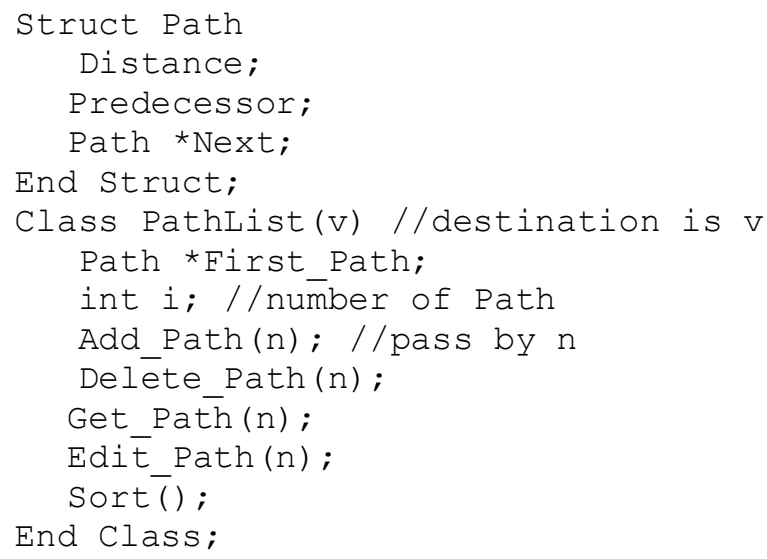


Our improved algorithm is shown in Algorithm 1. In Algorithm 1, the algorithm not only saves the shortest path, but also saves some valid paths from $S$ to vertex $v$ in PathList(v). Therefore, according to different requirements of Quality of Service (QoS) in the packet transmission, we can use several paths to transfer packets synchronously for decreasing the packet loss rate in the improved routing scheme.

Algorithm 1. Improved Bellman-Ford algorithm with multipath support

\section{/ / Step 1}

Determine Single Source $(G, S)$;

Distance $(\mathrm{s})=0$;

Predecessor $(\mathrm{s})=\mathrm{nil}$;

For each vertex $v$ in $G$ other than $s$

Distance $(v)=$ Infinity,

Predecessor $(v)=$ nil;

PathList (v) ->FirstPath. Distance

Infinity;

PathList $(v)->$ FirstPath. Predecessor = nil;

PathList $(v)->$ FirstPath $->$ Next $=$ NULL;

PathList $(v) \cdot i=0$;

Next;

/ / Step 2

For $i=1$ To $n-1$

For each edge $(u, v)$ in $G$

If $s$ connects to u directly Then

If PathList (v). Get_Path(u) =

True Then

$$
\text { PathList (v).Edit_Path (u); }
$$$$
\text { Else }
$$

PathList (v). Add_Path (u);

End If ;

End If;

If Distance $(v)>$ Distance $(u)+$

Weight (u, v) Then

Weight $(u, v)$; Distance $(v)=$ Distance $(u)+$

Predecessor $(v)=u$; End If;

Next;

PathList (v). Sort();

Next;

/ / Step 3

For each edge $(u, v)$ in $G$

If Distance $(v)>$ Distance $(u)+$

Weight (u, v) Then

Return False;

//This means that the graph contains a cycle of negative weight and the shortest paths are not well defined.

Else

Return True;

End If;

\section{UNIQUE LABEL FOR MULTIPATH ROUTING SCHEME}

In the multipath routing scheme, the receiver only receives the first packet of the same arrival packets. Because a packet is duplicated for the data transferring in several different paths in this scheme, there may be some inestimable errors in the application system in the receiver if the receiver accepts the same packets from the different paths. Therefore, after the receiver receives the first packet of the same arrival packets, it has to discard other repeated packets. For this purpose, we use a unique label to mark every packet in the sender before the packet is sent to the receiver in the multipath routing scheme.

The unique label can be defined either in the application layer or in the network layer. In the application layer, the unique label is defined when the packet is built in the application system, while the unique label is defined in the packet identification field in the network layer. In order to detect the uniqueness of the packet, every sender or receiver maintains a FIFO queue with constant length. The sender not only uses a new unique label to mark a packet before the packet is sent, but also saves this unique label to the FIFO queue. This new unique label is different from other unique labels in the FIFO queue of the sender. In the receiver, it compares the saved unique labels in the FIFO queue with the new unique label from the new arriving packet. If the new unique label is not equal to all the unique labels in FIFO queue, the receiver accepts this new arriving packet and saves this unique label to the FIFO queue. Otherwise, the receiver discards this new arriving packet if the new unique label is equal to any unique label in FIFO queue of the receiver. The purpose of the FIFO queue is to assure the uniqueness of the label for some time.

How to generate a unique label is also an important issue. In our study, a method which combines the physical address in the network adapter in the sender and the random number into the unique label is used. The reason why we use this method is that every network adapter has unique physical address. If the sender does not obtain the physical address of the network adapter, another unique label which combines the system time in the sender and the random number is used.

\section{PERFORMANCE ANALYSIS}

The NS2 simulation [14] for the performance analysis is used in our study. In this simulation scheme we simulate a wireless sensor network system which includes 11 nodes in NS2. In order to make the packet loss rate in the data transferring, every node keeps a distance from other nearby nodes. We set the node 1 as sender and the node 11 as receiver. The node 1 sends 10 UDP packets to the node 11 per second. The simulation topology is shown in Fig.1. In the simulation we use the minimum hops as the routing criteria. Fig. 2 shows the packet loss rate when the simulation system uses the traditional Bellman-Ford algorithm or the improved Bellman-Ford algorithm. In contrast to the traditional Bellman-Ford algorithm, the packet loss rate in the multipath routing scheme is lower obviously. This conclusion is similar to the result in the numerical analysis. To sum up, the real- 
time property is enhanced obviously based on our improved routing scheme in the industrial wireless sensor networks.

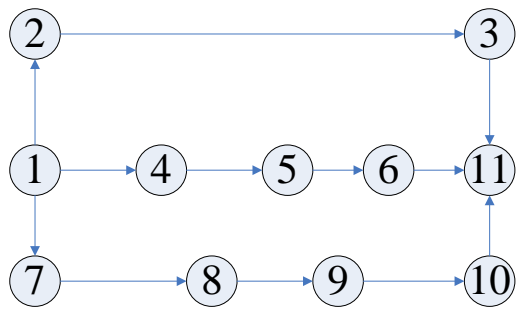

Figure 1. Simulation topology

\section{CONCLUSION}

A novel routing scheme is proposed to decrease the packet loss rate effectively in the industrial wireless sensor networks. This scheme is based on Bellman-Ford Algorithm. In the work principle, the scheme not only save the shortest path, but also save some other shorter paths for the multipath transmission in the Bellman-Ford algorithm. According to different requirements of QoS in data transferring, the scheme can use more than one path to send the same packets synchronously. We also testify the efficiency of the proposed routing scheme by the performance analysis. Therefore, this novel routing scheme can reduce the probability of data retransmission, and enhance the real-time property in the industrial wireless sensor networks.

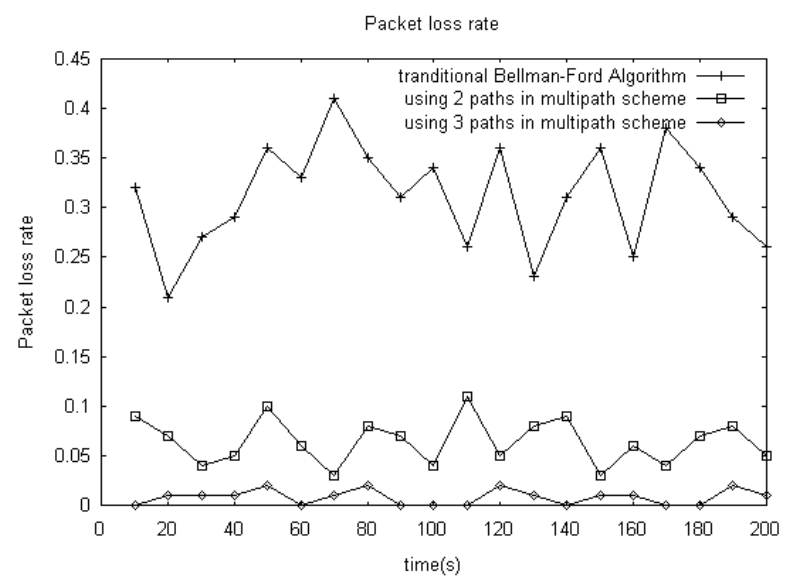

Figure 2. Packet loss rate in different schemes

\section{ACKNOWLEDGMENT}

The generous support of National Natural Science Foundation of China (11404150), Foundation of Jiangxi Educational Committee (GJJ150341, GJJ150311) and The Open Fund Project for Key Laboratory of Photoelectronics and Telecommunication of Jiangxi are gratefully acknowledged.

\section{REFERENCES}

[1] Mahmood, M. Adeel, W. K. G. Seah, and I. Welch. "Reliability in wireless sensor networks: A survey and challenges ahead," Computer Networks, 2015, 79, pp.166-187.

[2] D. Zhang, G. Li, K. Zheng, X .Ming, and Z. H. Pan, "An energybalanced routing method based on forward-aware factor for wireless sensor networks," IEEE transactions on industrial informatics, 2014 10(1), pp.766-773.

[3] Hammoudeh, Mohammad, and R. Newman, "Adaptive routing in wireless sensor networks: QoS optimisation for enhanced application performance," Information Fusion, 2015, 22, pp.3-15.

[4] Y. Yao, Q. Cao, and A. V. Vasilakos, "EDAL: An energy-efficient, delay-aware, and lifetime-balancing data collection protocol for heterogeneous wireless sensor networks," IEEE/ACM Transactions on Networking,2015, 23(3), pp. 810-823.

[5] C. E. Perkins and E. M. Royer, "Ad hoc On-Demand Distance Vector Routing," In 2nd IEEE Workshop on Mobile Computing Systems and Applications, New Orleans, LA, USA,1999.

[6] S. Murthy and J. J. Garcia-Luna-Aceves, "An efficient routing protocol for wireless networks," Mobile Networks and Applications 1: 183-197, 1996.

[7] G. Pei, M. Gerla, X. Hong, and C. C. Chiang, "Wireless Hierarchical Routing Protocol with Group Mobility (WHIRL)," In IEEE Wireless Communications and Networking Conference, New Orleans, LA, USA, 1999

[8] J. J. Garcia-Luna-Aceves and M. Spohn, "Source-Tree Routing in Wireless Networks," In 7th International Conference on Network Protocols, Toronto, Canada, 1999.

[9] C. Chiang, H. Wu, W. Liu, M. Gerla, "Routing in Clustered Multihop, Mobile Wireless Networks with Fading Channel," In Proceeding of IEEE Singapore International Conference on Networks, Singapore, 1997.

[10] C. Zhu and M. S. Corson, "QoS routing for mobile ad hoc networks," In Proceeding of IEEE INFOCOM2002, New York, USA, 2002.

[11] V. Park and S. Corson, "Temporally-Ordered Routing Algorithm (TORA)," http://www.ietf.org/proceedings/53/I-D/draft-ietf-manettora-spec-04.txt, 2001.

[12] K. Akkaya and M. Younis, "A survey on routing protocols for wireless sensor networks," Ad Hoc Networks 3:325-349, 2005.

[13] R. Bellman and L. Ford, "On a routing problem. Quarterly of Applied Mathematics," 1958, 16, pp.87-90.

[14] Information Science Institute, "NS2 - The network simulator," http:// http://www.isi.edu/nsnam/ns/, 2006. 